\title{
Analytical and numerical methods of converting Cartesian to ellipsoidal coordinates
}

DOI: https://doi.org/10.1515/jogs-2020-0126

Received March 8, 2021; accepted September 27, 2021

\begin{abstract}
In this work, two analytical and two numerical methods of converting Cartesian to ellipsoidal coordinates of a point in space are presented. After slightly modifying a well-known exact analytical method, a new exact analytical method is developed. Also, two well-known numerical methods, which were developed for points exactly on the surface of a triaxial ellipsoid, are generalized for points in space. The four methods are validated with numerical experiments using an extensive set of points for the case of the Earth. Then, a theoretical and a numerical comparative assessment of the four methods is made. Furthermore, the new exact analytical method is applied for an almost oblate spheroid and for the case of the Moon and the results are compared. We conclude that, the generalized Panou and Korakitis' numerical method, starting with approximate values from the new exact analytical method, is the best choice in terms of accuracy of the resulting ellipsoidal coordinates.
\end{abstract}

Keywords: exact method, Laplace's equation, orthogonal net, triaxial ellipsoid

\section{Introduction}

Ellipsoidal coordinates, as a triply orthogonal coordinate system, have the advantage of decomposing the Laplace partial differential equation into separable functions. Thus, these coordinates are particularly valuable in potential theory. Also, the coordinate surface of a triaxial ellipsoid, that fits the physical boundary of a problem, helps to express a boundary condition in a simple way. For instance, one may use ellipsoidal coordinates in the gravity field which has a triaxial ellipsoid as a level surface. Other examples of the use of these coordinates can be found in Dassios (2012).

G. Panou: Department of Surveying Engineering, National Technical University of Athens, 15780 Athens, Greece, E-mail: geopanou@survey.ntua.gr

R. Korakitis: Department of Surveying Engineering, National Technical University of Athens, 15780 Athens
Usually, three variants of ellipsoidal coordinates, namely $\left(s_{1}, s_{2}, s_{3}\right),(\rho, \mu, v)$ and $(u, \beta, \lambda)$ are used in the literature. These variants are presented e.g. by Tabanov (1999), Dassios (2012) and Panou (2014). However, the coordinates $(u, \beta, \lambda)$ are the only ones with invariant intervals of variation as the system transforms to the oblate spheroidal system (Dassios 2012). In other words, using the ellipsoidal coordinates $(u, \beta, \lambda)$ we avoid the degeneracy of the coordinates $s_{3}$ and $v$, as the triaxial ellipsoid approaches an oblate spheroid. This is of special importance for geodetic applications related to the Earth, so we will study this variant only in the present work.

The Cartesian coordinates $(x, y, z)$ of a point in space can be obtained from the ellipsoidal coordinates (ellipsoidal parameter $u \in[0,+\infty)$, ellipsoidal latitude $\beta \in$ $\left[-\frac{\pi}{2},+\frac{\pi}{2}\right]$ and ellipsoidal longitude $\left.\lambda \in(-\pi,+\pi]\right)$, introduced by Tabanov (1999) and also presented in Panou (2014), by

$$
\begin{gathered}
x=\left(u^{2}+E_{x}^{2}\right)^{\frac{1}{2}} B^{\frac{1}{2}} \cos \lambda \\
y=\left(u^{2}+E_{y}^{2}\right)^{\frac{1}{2}} \cos \beta \sin \lambda \\
z=u \sin \beta L^{\frac{1}{2}}
\end{gathered}
$$

where

$$
B=\cos ^{2} \beta+\frac{E_{e}^{2}}{E_{X}^{2}} \sin ^{2} \beta
$$

and

$$
L=1-\frac{E_{e}^{2}}{E_{X}^{2}} \cos ^{2} \lambda
$$

while

$$
E_{x}=\left(a_{x}^{2}-b^{2}\right)^{\frac{1}{2}}, E_{y}=\left(a_{y}^{2}-b^{2}\right)^{\frac{1}{2}}, E_{e}=\left(a_{x}^{2}-a_{y}^{2}\right)^{\frac{1}{2}},
$$

are the linear eccentricities (obviously $E_{e}^{2}=E_{x}^{2}-E_{y}^{2}$ ) of the triaxial ellipsoid $(u=b)$ :

$$
\frac{x^{2}}{a_{x}^{2}}+\frac{y^{2}}{a_{y}^{2}}+\frac{z^{2}}{b^{2}}=1,0<b<a_{y}<a_{x}
$$

where $a_{x}, a_{y}$ and $b$ are its three semi-axes.

The geometrical interpretation of these ellipsoidal coordinates is given by Panou (2014). Specifically, the ellipsoidal parameter $u$ is the polar semi-axis of the confocal el- 
lipsoid which passes through the point $(x, y, z)$. The ellipsoidal latitude $\beta$ represents the inclination of the asymptotes of the confocal principal hyperbola on the plane $x=$ 0 . The ellipsoidal longitude $\lambda$ represents the inclination of the asymptotes of the confocal principal hyperbola on the plane $z=0$. In the case of an oblate spheroid, where $a_{x}=a_{y} \equiv a$, i.e. $E_{x}=E_{y} \equiv E$ and $E_{e}=0$, Eqs. (1)(3) reduce to well-known expressions (see Heiskanen and Moritz 1967).

In the case where $u=b$, i.e. for points located on the surface of a triaxial ellipsoid, Eqs. (1)-(3) reduce to wellknown expressions introduced by Jacobi (1839). These curvilinear ellipsoidal coordinates $(\beta, \lambda)$ constitute an orthogonal net of curves on the surface of a triaxial ellipsoid, therefore they eliminate cumbersome mathematical expressions for problems like the study of geodesics (e.g. Panou and Korakitis 2019) and for simple determinations, such as the surface area. Thus, the problem of converting the Cartesian coordinates $(x, y, z)$ of a point on the surface of a triaxial ellipsoid to the corresponding ellipsoidal coordinates $(\beta, \lambda)$ is of special importance. For the solution of this problem, an exact analytical and a numerical method were developed by Panou and Korakitis (2019). In that work, an extensive test set was used, in order to evaluate the performance of the two methods in double and quad precision. These numerical validations using points on the surface of the triaxial ellipsoid gave results better than $1 \mathrm{~mm}$ for double and $1 \mathrm{~nm}$ for quad precision. We should also mention another numerical method which was developed by Bektas (2015) and was used in the works of Florinsky (2018) and Pędzich (2019).

In the present work, we attempt to solve the problem of converting the Cartesian coordinates $(x, y, z)$ of a point in space to the corresponding ellipsoidal coordinates $(u, \beta, \lambda)$, using two analytical and two numerical methods. In Section 2.1., we present a modification of the exact analytical method described by Panou (2014) with a change of variable in the relevant cubic equation. In Section 2.2., a new exact analytical method is developed. In Section 3., the numerical methods, developed by Bektas (2015) and Panou and Korakitis (2019), are generalized for points in space (not only located on the surface). Thus, the coordinate $u$ is needed in the development of the relevant algorithms. Finally, both a theoretical and a numerical comparative assessment of the four methods is made, mainly in terms of the accuracy of the resulting ellipsoidal coordinates.

In the remainder of this section, we present the required mathematical formulas for the development of the methods. Also, for simplicity and without loss of generality, we always assume $x \geq 0, y \geq 0$ and $z \geq 0$. Hence, after the conversion, the conventions with regard to the proper quadrant for the $\beta$ and $\lambda$ need to be applied from the signs of $x, y$, and $z$. Furthermore, in order to ensure the precision of the numerical computations, we use functions which are continuous and without singularities within their domain of use and beyond their endpoints if possible, e.g. using absolute values and variants of functions for different domains of space.

From Eqs. (2) and (3), we obtain

$$
\tan ^{2} \beta=M \frac{\sin ^{2} \lambda}{L}
$$

and from Eqs. (1) and (2)

$$
\tan ^{2} \lambda=P \frac{B}{\cos ^{2} \beta}
$$

where

$$
M=\frac{z^{2}}{y^{2}} \cdot \frac{u^{2}+E_{y}^{2}}{u^{2}}
$$

and

$$
P=\frac{y^{2}}{x^{2}} \cdot \frac{u^{2}+E_{x}^{2}}{u^{2}+E_{y}^{2}}
$$

Inverting Eqs. (8)-(11) results to

$$
\cot ^{2} \beta=N \frac{L}{\sin ^{2} \lambda}
$$

and

$$
\cot ^{2} \lambda=Q \frac{\cos ^{2} \beta}{B}
$$

where

$$
N=M^{-1}=\frac{y^{2}}{z^{2}} \cdot \frac{u^{2}}{u^{2}+E_{y}^{2}}
$$

and

$$
Q=P^{-1}=\frac{x^{2}}{y^{2}} \cdot \frac{u^{2}+E_{y}^{2}}{u^{2}+E_{x}^{2}}
$$

Hence, from Eqs. (8) and (9), we have

$$
\begin{gathered}
\beta=\tan ^{-1}\left[\left(\frac{M}{L}\right)^{\frac{1}{2}} \sin \lambda\right] \\
\lambda=\tan ^{-1}\left[\frac{(P B)^{\frac{1}{2}}}{\cos \beta}\right]
\end{gathered}
$$

and from Eqs. (12) and (13)

$$
\begin{gathered}
\beta=\frac{\pi}{2}-\tan ^{-1}\left[\frac{(N L)^{\frac{1}{2}}}{\sin \lambda}\right] \\
\lambda=\frac{\pi}{2}-\tan ^{-1}\left[\left(\frac{Q}{B}\right)^{\frac{1}{2}} \cos \beta\right]
\end{gathered}
$$




\section{Analytical methods}

\subsection{Case of a triaxial ellipsoid only}

The ellipsoidal coordinates $(u, \beta, \lambda)$ can be obtained from the Cartesian coordinates $(x, y, z)$ of a point in space by solving the following cubic equation in $t$

$$
t^{3}+c_{2} t^{2}+c_{1} t+c_{0}=0
$$

where

$$
\begin{gathered}
c_{2}=E_{x}^{2}+E_{y}^{2}-x^{2}-y^{2}-z^{2} \\
c_{1}=E_{x}^{2} E_{y}^{2}-E_{y}^{2} x^{2}-E_{x}^{2} y^{2}-\left(E_{x}^{2}+E_{y}^{2}\right) z^{2} \\
c_{0}=-E_{x}^{2} E_{y}^{2} z^{2}
\end{gathered}
$$

Comparing Eq. (20) with the corresponding equation presented by Panou (2014), the two cubic equations are related with the transformation $s=t-b^{2}$. Here, the coefficients are expressed in terms of the linear eccentricities and thus keep the magnitude to a moderate size. Also, Eq. (20) has three real roots, which are distributed as follows

$$
-E_{x}^{2} \leq t_{3} \leq-E_{y}^{2} \leq t_{2} \leq 0 \leq t_{1}<+\infty
$$

and can be represented using trigonometric functions as

$$
\begin{gathered}
t_{1}=2 p^{\frac{1}{2}} \cos \left(\frac{\omega}{3}\right)-\frac{c_{2}}{3} \\
t_{2}=2 p^{\frac{1}{2}} \cos \left(\frac{\omega-2 \pi}{3}\right)-\frac{c_{2}}{3} \\
t_{3}=2 p^{\frac{1}{2}} \cos \left(\frac{\omega-4 \pi}{3}\right)-\frac{c_{2}}{3}
\end{gathered}
$$

where

$$
\begin{gathered}
p=\frac{c_{2}^{2}-3 c_{1}}{9} \\
q=\frac{9 c_{1} c_{2}-27 c_{0}-2 c_{2}^{3}}{54}
\end{gathered}
$$

and

$$
\omega=\cos ^{-1}\left(\frac{q}{p^{\frac{3}{2}}}\right), \text { if } q<p^{\frac{3}{2}} \text { else } \omega=0
$$

Then, the ellipsoidal coordinates $(u, \beta, \lambda)$ are given by

$$
\begin{gathered}
u=\left(t_{1}\right)^{\frac{1}{2}} \\
\beta=\tan ^{-1}\left(\left|\frac{-t_{2}}{E_{y}^{2}+t_{2}}\right|\right)^{\frac{1}{2}}, \text { if }\left|-t_{2}\right| \leq\left|E_{y}^{2}+t_{2}\right|
\end{gathered}
$$

or

$$
\beta=\frac{\pi}{2}-\tan ^{-1}\left(\left|\frac{E_{y}^{2}+t_{2}}{-t_{2}}\right|\right)^{\frac{1}{2}}, \text { if }\left|-t_{2}\right|>\left|E_{y}^{2}+t_{2}\right|
$$

and

$$
\lambda=\tan ^{-1}\left(\left|\frac{-E_{y}^{2}-t_{3}}{E_{x}^{2}+t_{3}}\right|\right)^{\frac{1}{2}}, \text { if }\left|-E_{y}^{2}-t_{3}\right| \leq\left|E_{x}^{2}+t_{2}\right|
$$

or

$$
\lambda=\frac{\pi}{2}-\tan ^{-1}\left(\left|\frac{E_{x}^{2}+t_{3}}{-E_{y}^{2}-t_{3}}\right|\right)^{\frac{1}{2}}, \text { if }\left|-E_{y}^{2}-t_{3}\right|>\left|E_{x}^{2}+t_{3}\right|
$$

In the case of an oblate spheroid, where $E_{x}=E_{y} \equiv E$ and therefore $t_{3}=-E^{2}$, the ellipsoidal longitude cannot be computed either from Eq. (34) or Eq. (35). Therefore, we cannot avoid the degeneracy of the variable $t_{3}$ for an oblate spheroid, thus Eq. (34) or Eq. (35) produce inaccurate results in the case of an almost oblate spheroid. In addition, the operations for the computation of ellipsoidal latitude and longitude lead to a loss of precision for points near the principal planes $(x=0$ or $y=0$ or $z=0$ ). All these reasons constitute the motivation for the development of the following analytical method which uses different mathematical formulas for such computations. However, for the development of all other methods of converting Cartesian to ellipsoidal coordinates which will be presented, the ellipsoidal parameter $u$ is considered known, as computed from Eq. (31).

\subsection{Case of a triaxial ellipsoid and an oblate spheroid}

The ellipsoidal parameter $u$ now being known, eliminating $\lambda$ between Eqs. (12) and (13) we obtain a quadratic equation for $\cot ^{2} \beta$, whose solution is

$$
\beta=\frac{\pi}{2}-\tan ^{-1}\left\{\frac{1}{2}\left[W+\left(W^{2}+4 \frac{E_{e}^{2}}{E_{x}^{2}} N\right)^{\frac{1}{2}}\right]\right\}^{\frac{1}{2}}
$$

where

$$
W=N+\frac{E_{y}^{2}}{E_{x}^{2}} \cdot \frac{x^{2}}{z^{2}} \cdot \frac{u^{2}}{u^{2}+E_{x}^{2}}-\frac{E_{e}^{2}}{E_{x}^{2}}
$$

Also, eliminating $\beta$ between Eqs. (8) and (9) we obtain a quadratic equation for $\tan ^{2} \lambda$, whose solution is

$$
\lambda=\tan ^{-1}\left\{\frac{1}{2}\left[U+\left(U^{2}+4 \frac{E_{y}^{2}}{E_{x}^{2}} P\right)^{\frac{1}{2}}\right]\right\}^{\frac{1}{2}}
$$

where

$$
U=P+\frac{E_{e}^{2}}{E_{x}^{2}} \cdot \frac{z^{2}}{x^{2}} \cdot \frac{u^{2}+E_{x}^{2}}{u^{2}}-\frac{E_{y}^{2}}{E_{x}^{2}}
$$

Finally, eliminating $\beta$ between Eqs. (8) and (13) we obtain a quadratic equation for $\cot ^{2} \lambda$, whose solution is

$$
\lambda=\frac{\pi}{2}-\tan ^{-1}\left\{\frac{1}{2}\left[V+\left(V^{2}+4 \frac{E_{x}^{2}}{E_{y}^{2}} Q\right)^{\frac{1}{2}}\right]\right\}^{\frac{1}{2}}
$$


where

$$
V=Q-\frac{E_{e}^{2}}{E_{y}^{2}} M-\frac{E_{x}^{2}}{E_{y}^{2}}
$$

Hence, starting from the given Cartesian coordinates and the semi-axes of the triaxial ellipsoid, we compute the ellipsoidal parameter $u$ from Eq. (31) and the quantities $M$, $N, P$ and $Q$ from Eqs. (10), (14), (11) and (15), respectively. Then, the algorithm proceeds as follows:

If $z \leq y \leq x$, we first compute $\lambda$ from Eq. (38) and then $\beta$ from Eq. (16). The case $y=0$ (i.e. $x$-axis) is excluded since $\beta=\lambda=0$. Also, if $z \leq y>x$, we first compute $\lambda$ from Eq. (40) and then $\beta$ from Eq. (16).

If $z>y \geq x$, we first compute $\beta$ from Eq. (36) and then $\lambda$ from Eq. (19). The case $y=0$ (i.e. $z$-axis) is excluded since $\beta=\lambda=\frac{\pi}{2}$. Also, if $z>y<x$, we compute $\beta$ from Eq. (36) and $\lambda$ from Eq. (38).

\subsubsection{Case of an oblate spheroid}

In the case of an oblate spheroid, where $E_{x}=E_{y} \equiv E$, the ellipsoidal coordinates are computed by the well-known expressions (e.g. Heiskanen and Moritz 1967)

$$
u=\left\{\frac{1}{2}\left[R+\left(R^{2}+4 E^{2} z^{2}\right)^{\frac{1}{2}}\right]\right\}^{\frac{1}{2}}
$$

where

$$
R=x^{2}+y^{2}+z^{2}-E^{2}
$$

while $\beta$ is given by

$\beta=\tan ^{-1}\left[\frac{z}{u} \cdot\left(\frac{u^{2}+E^{2}}{x^{2}+y^{2}}\right)^{\frac{1}{2}}\right]=\frac{\pi}{2}-\tan ^{-1}\left[\frac{u}{z} \cdot\left(\frac{x^{2}+y^{2}}{u^{2}+E^{2}}\right)^{\frac{1}{2}}\right]$

and $\lambda$ by

$$
\lambda=\tan ^{-1}\left(\frac{y}{x}\right)=\frac{\pi}{2}-\tan ^{-1}\left(\frac{x}{y}\right)
$$

It is worth mentioning that Eqs. (31) and (42), although different in form, are equivalent in the case of an oblate spheroid, where $E_{x}=E_{y} \equiv E$ and $E_{e}=0$. Furthermore, Eqs. (36), (38) and (40) after the necessary simplifications, reduce to Eqs. (44) and (45).

\section{Numerical methods}

\subsection{Generalized Bektas' method}

Knowing the Cartesian coordinates $(x, y, z)$ of a point in space and the ellipsoidal parameter $u$, hence also the quantities $M, N, P$ and $Q$, Eqs. (16) and (18) are rewritten as

$$
\begin{aligned}
& \beta_{i+1}=\tan ^{-1}\left[\left(\frac{M}{L_{i}}\right)^{\frac{1}{2}} \sin \lambda_{i}\right] \\
& \beta_{i+1}=\frac{\pi}{2}-\tan ^{-1}\left[\frac{\left(N L_{i}\right)^{\frac{1}{2}}}{\sin \lambda_{i}}\right]
\end{aligned}
$$

and Eqs. (17) and (19) as

$$
\begin{gathered}
\lambda_{i+1}=\tan ^{-1}\left[\frac{\left(P B_{i+1}\right)^{\frac{1}{2}}}{\cos \beta_{i+1}}\right] \\
\lambda_{i+1}=\frac{\pi}{2}-\tan ^{-1}\left[\left(\frac{Q}{B_{i+1}}\right)^{\frac{1}{2}} \cos \beta_{i+1}\right]
\end{gathered}
$$

where

$$
B_{i+1}=\cos ^{2} \beta_{i+1}+\frac{E_{e}^{2}}{E_{x}^{2}} \sin ^{2} \beta_{i+1}
$$

and

$$
L_{i}=1-\frac{E_{e}^{2}}{E_{x}^{2}} \cos ^{2} \lambda_{i}
$$

where $i=0,1,2, \ldots$ and an approximate initial value of ellipsoidal longitude $\lambda_{0}$ is required. Then, the algorithm proceeds as follows:

If $z \leq y \leq x$, we first compute $\beta$ from Eq. (46) and then $\lambda$ from Eq. (48). The case $y=0$ (i.e. $x$-axis) is excluded since $\beta=\lambda=0$. Also, if $z \leq y>x$, we first compute $\beta$ from Eq. (46) and then $\lambda$ from Eq. (49).

If $z>y \geq x$, we first compute $\beta$ from Eq. (47) and then $\lambda$ from Eq. (49). The case $y=0$ (i.e. $z$-axis) is excluded since $\beta=\lambda=\frac{\pi}{2}$. Also, if $z>y<x$, we first compute $\beta$ from Eq. (47) and then $\lambda$ from Eq. (48). Thus, the $x z$-plane is excluded and the method fails to give a solution.

In all cases, the process is repeated until the values $d \beta=\left|\beta_{i+1}-\beta_{i}\right|$ and $d \lambda=\left|\lambda_{i+1}-\lambda_{i}\right|$ become negligibly small. We note that, the iterative method described in Bektas (2015) uses only Eqs. (46) and (48) and approximate values for both coordinates $\beta_{0}$ and $\lambda_{0}$.

\subsection{Generalized Panou Korakitis' method}

Considering the Cartesian coordinates $(x, y, z)$ of a point in space as measurements, the application of the method of least squares (e.g. Ghilani and Wolf 2006) requires writing of Eqs. (1)-(3) in the form

$$
\begin{gathered}
\left(u^{2}+E_{x}^{2}\right)^{\frac{1}{2}} B^{\frac{1}{2}} \cos \lambda=x+v_{x} \\
\left(u^{2}+E_{y}^{2}\right)^{\frac{1}{2}} \cos \beta \sin \lambda=y+v_{y} \\
u \sin \beta L^{\frac{1}{2}}=z+v_{z}
\end{gathered}
$$


which, in linear approximation, can be represented as

$$
\mathbf{J}\left[\begin{array}{l}
d \beta \\
d \lambda
\end{array}\right]=\boldsymbol{\delta} \mathbf{1}+\boldsymbol{v}
$$

where the $3 \times 2$ Jacobian matrix is

$$
\mathbf{J}=\left[\begin{array}{ll}
\frac{\partial x}{\partial \beta} & \frac{\partial x}{\partial \lambda} \\
\frac{\partial y}{\partial \beta} & \frac{\partial y}{\partial \lambda} \\
\frac{\partial z}{\partial \beta} & \frac{\partial z}{\partial \lambda}
\end{array}\right]
$$

with elements

$$
\begin{gathered}
\frac{\partial x}{\partial \beta}=-\left(u^{2}+E_{x}^{2}\right)^{\frac{1}{2}} \frac{E_{y}^{2}}{E_{x}^{2}} \cdot \frac{\sin (2 \beta)}{2 B^{\frac{1}{2}}} \cos \lambda \\
\frac{\partial y}{\partial \beta}=-\left(u^{2}+E_{y}^{2}\right)^{\frac{1}{2}} \sin \beta \sin \lambda \\
\frac{\partial z}{\partial \beta}=u \cos \beta L^{\frac{1}{2}} \\
\frac{\partial x}{\partial \lambda}=-\left(u^{2}+E_{x}^{2}\right)^{\frac{1}{2}} B^{\frac{1}{2}} \sin \lambda \\
\frac{\partial y}{\partial \lambda}=\left(u^{2}+E_{y}^{2}\right)^{\frac{1}{2}} \cos \beta \cos \lambda \\
\frac{\partial z}{\partial \lambda}=u \frac{E_{e}^{2}}{E_{x}^{2}} \sin \beta \frac{\sin (2 \lambda)}{2 L^{\frac{1}{2}}}
\end{gathered}
$$

which are computed from the known ellipsoidal parameter $u$ and approximate ellipsoidal coordinates $\beta_{0}$ and $\lambda_{0}$. The $3 \times 1$ vector

$$
\boldsymbol{\delta} \mathbf{1}=\left[\begin{array}{c}
x-x_{0} \\
y-y_{0} \\
z-z_{0}
\end{array}\right]
$$

is computed from the Cartesian coordinates $\left(x_{0}, y_{0}, z_{0}\right)$ of the approximate ellipsoidal coordinates $\beta_{0}$ and $\lambda_{0}$ and $\boldsymbol{v}$ is a $3 \times 1$ vector of residuals. The updated values of the approximate ellipsoidal coordinates are

$$
\left[\begin{array}{l}
\beta \\
\lambda
\end{array}\right]=\left[\begin{array}{l}
\beta_{0} \\
\lambda_{0}
\end{array}\right]+\left[\begin{array}{l}
d \beta \\
d \lambda
\end{array}\right]
$$

where the solution vector is given by

$$
\left[\begin{array}{l}
d \beta \\
d \lambda
\end{array}\right]=\mathbf{N}^{-1} \mathbf{J}^{\mathbf{T}} \boldsymbol{\delta} \mathbf{1}
$$

while

$$
\mathbf{N}=\mathbf{J}^{\mathbf{T}} \mathbf{J}=\left[\begin{array}{ll}
n_{11} & n_{12} \\
n_{21} & n_{22}
\end{array}\right]
$$

and hence

$$
\mathbf{N}^{-1}=\frac{1}{n_{11} n_{22}-n_{12} n_{21}}\left[\begin{array}{cc}
n_{22} & -n_{12} \\
-n_{21} & n_{11}
\end{array}\right]
$$

We note that the determinant of the matrix $N$ equals zero only at the umbilical points, where $\beta=\frac{\pi}{2}$ and $\lambda=0$, by definition. The process is repeated until the corrections $|d \beta|$ and $|d \lambda|$ become negligibly small.

\section{Numerical tests and comparisons}

\subsection{Test set}

In order to validate the four methods of conversion presented above and to evaluate their performance, we used an extensive test set of points. This is a set of 7023 points in space, distributed into thirteen groups, as described in Table 1. For the ellipsoidal parameter $u$, three representative values were chosen: $u=\frac{b}{2}$ (inside ellipsoid), $u=b$ (on the ellipsoid) and $u=2 b$ (outside ellipsoid). Also, for simplicity and without loss of generality, $\beta$ and $\lambda$ were chosen in $\left[0^{\circ}, 90^{\circ}\right]$.

Models of three different bodies were considered: a triaxial ellipsoid like the Earth, with $a_{x}=6378172 \mathrm{~m}$, $a_{y}=6378102 \mathrm{~m}$ and $b=6356752 \mathrm{~m}$ (Panou et al. 2020), an almost oblate spheroid $a_{x}=6378137.001 \mathrm{~m}, a_{y}=$ $6378137 \mathrm{~m}$ and $b=6356752 \mathrm{~m}$ and a triaxial ellipsoid like the Moon, with $a_{x}=1738056 \mathrm{~m}, a_{y}=1737843 \mathrm{~m}$ and $b=1735485 \mathrm{~m}$ (Iz 2009). The corresponding Cartesian coordinates for each body and for any point were computed using Eqs. (1)-(3), keeping 27 significant digits in the results.

All algorithms were implemented in $\mathrm{C}++$ and compiled using the GNU Compiler Collection version 9.2 at optimization level 3, running under the Debian Linux operating system. We employed the open-source "libquadmath”, the GCC Quad-Precision Math Library, which provides a precision of 33 digits (referred to as "quad"). In contrast, the long double data type (referred to simply as "double") maps to the IEEE-754 80-bit extended precision type on this platform and provides a precision of 18 digits. The codes were executed on a personal computer running a 64-bit Linux Debian operating system. The main characteristics of the hardware were: AMD Ryzen $74800 \mathrm{H} \mathrm{CPU}$ (with 8 cores and clocked at $2.9 \mathrm{GHz}$ ) and $16 \mathrm{~GB}$ of RAM.

In addition, we note that all figures, presented in the following sections, were produced using the MATLAB software package.

Furthermore, we added a test set of 300 points in space with $\beta=\lambda=45^{\circ}$ and values of $u$ from $100 \mathrm{~km}$ to $30000 \mathrm{~km}$, with a step size $100 \mathrm{~km}$ (Group 14), in order to validate the performance of the methods with respect to a wider range of values of the ellipsoidal parameter $u$.

\section{Results}

All four methods were tested, in the case of the Earth, using double and quad precision and the Cartesian coordi- 
Table 1. Description of the points in the test set (total 7023 points)

\begin{tabular}{|c|c|c|c|c|c|c|}
\hline \multicolumn{2}{|c|}{ Group } & Case & $u$ & $\beta$ & $\lambda$ & Number \\
\hline 1 & G & $1^{\text {st }}$ octant & $\frac{b}{2}, b, 2 b$ & $5^{\circ}-85^{\circ}$ every $5^{\circ}$ & $5^{\circ}-85^{\circ}$ every $5^{\circ}$ & 867 \\
\hline 2 & A1 & $x y-$ plane & $\frac{b}{2}, b, 2 b$ & $0^{\circ}$ & $5^{\circ}-90^{\circ}$ every $5^{\circ}$ & 54 \\
\hline 3 & A2.1 & \multirow{2}{*}{$x z-$ plane } & $\frac{b}{2}, b, 2 b$ & $0^{\circ}-90^{\circ}$ every $5^{\circ}$ & $0^{\circ}$ & 57 \\
\hline 4 & $\mathrm{~A} 2.2$ & & $\frac{b}{2}, b, 2 b$ & $90^{\circ}$ & $5^{\circ}-90^{\circ}$ every $5^{\circ}$ & 54 \\
\hline 5 & A3 & $y z-$ plane & $\frac{b}{2}, b, 2 b$ & $5^{\circ}-85^{\circ}$ every $5^{\circ}$ & $90^{\circ}$ & 51 \\
\hline 6 & B1 & near $x y-$ plane & $\frac{b}{2}, b, 2 b$ & $\begin{array}{c}1.0^{\circ}-0.0 \ldots 1^{\circ} \text { up to } 14 \\
\text { decimals }\end{array}$ & $5^{\circ}-90^{\circ}$ every $5^{\circ}$ & 810 \\
\hline 7 & B2.1 & \multirow{2}{*}{ near $x z-$ plane } & $\frac{b}{2}, b, 2 b$ & $0^{\circ}-90^{\circ}$ every $5^{\circ}$ & $\begin{array}{c}1.0^{\circ}-0.0 \ldots 1^{\circ} \text { up to } 14 \\
\text { decimals }\end{array}$ & 855 \\
\hline 8 & B2.2 & & $\frac{b}{2}, b, 2 b$ & $\begin{array}{c}89.0^{\circ}-89.9 \ldots 9^{\circ} \text { up to } \\
14 \text { decimals }\end{array}$ & $5^{\circ}-90^{\circ}$ every $5^{\circ}$ & 810 \\
\hline 9 & B3 & near $y z-$ plane & $\frac{b}{2}, b, 2 b$ & $5^{\circ}-85^{\circ}$ every $5^{\circ}$ & $\begin{array}{c}89.0^{\circ}-89.9 \ldots 9^{\circ} \text { up to } \\
14 \text { decimals }\end{array}$ & 765 \\
\hline 10 & $\mathrm{C} 1$ & near $x-$ axis & $\frac{b}{2}, b, 2 b$ & $\begin{array}{c}1.0^{\circ}-0.0 \ldots 1^{\circ} \text { up to } 14 \\
\text { decimals }\end{array}$ & $\begin{array}{c}1.0^{\circ}-0.0 \ldots 1^{\circ} \text { up to } 14 \\
\text { decimals }\end{array}$ & 675 \\
\hline 11 & $\mathrm{C} 2$ & near $y-$ axis & $\frac{b}{2}, b, 2 b$ & $\begin{array}{c}1.0^{\circ}-0.0 \ldots 1^{\circ} \text { up to } 14 \\
\text { decimals }\end{array}$ & $\begin{array}{c}89.0^{\circ}-89.9 \ldots 9^{\circ} \text { up to } \\
14 \text { decimals }\end{array}$ & 675 \\
\hline 12 & C3 & near $z-$ axis & $\frac{b}{2}, b, 2 b$ & $\begin{array}{c}89.0^{\circ}-89.9 \ldots 9^{\circ} \text { up to } \\
14 \text { decimals }\end{array}$ & $\begin{array}{c}89.0^{\circ}-89.9 \ldots 9^{\circ} \text { up to } \\
14 \text { decimals }\end{array}$ & 675 \\
\hline 13 & D & near umbilic & $\frac{b}{2}, b, 2 b$ & $\begin{array}{c}89.0^{\circ}-89.9 \ldots 9^{\circ} \text { up to } \\
14 \text { decimals }\end{array}$ & $\begin{array}{c}1.0^{\circ}-0.0 \ldots 1^{\circ} \text { up to } 14 \\
\text { decimals }\end{array}$ & 675 \\
\hline
\end{tabular}

nates $x, y$ and $z$ as input data. From the resulting $u^{\prime}, \beta^{\prime}$ and $\lambda^{\prime}$ at any point, we computed the differences $\delta u=u-u^{\prime}$, $\delta \beta=\beta-\beta^{\prime}$ and $\delta \lambda=\lambda-\lambda^{\prime}$, and recorded the maximum absolute values, i.e. $\max |\delta u|, \max |\delta \beta|$ and $\max |\delta \lambda|$ for every Group. Furthermore, because Eqs. (1)-(3) are numerically stable, the results at any point were checked by comparing the resulting Cartesian coordinates $\left(x^{\prime}, y^{\prime}, z^{\prime}\right)$ with the original Cartesian coordinates $(x, y, z)$ by the simple Euclidean distance formula

$$
\delta r=\left[\left(x-x^{\prime}\right)^{2}+\left(y-y^{\prime}\right)^{2}+\left(z-z^{\prime}\right)^{2}\right]^{\frac{1}{2}}
$$

For every Group, we recorded the maximum value, i.e. $\max \delta r$. We remark that only the quantity $\delta r$ can be computed when starting with knowledge of the Cartesian coordinates.

Additionally, in the numerical methods we used approximate values of the coordinates $\beta$ and $\lambda$ from the analytical method of Section 2.1. and we recorded the mean and the maximum value of iterations $i$, which were needed. As criterion of convergence of the $d \beta$ and $d \lambda$, the values $10^{-19} \mathrm{rad}$ and $10^{-34} \mathrm{rad}$, for double and quad precision, respectively, were used.

All results are presented in Tables 2 to 5 , where lengths $\delta u$ and $\delta r$ are expressed in meters and angular quantities $\delta \beta$ and $\delta \lambda$ in arcseconds. Whenever the value of a quantity appears as " 0 ", this implies that it is less than the corresponding machine precision. Since the values of $\max |\delta u|$ are common in all methods, they appear in Table 2 only.

From the values of $\max |\delta u|$ presented in Table 2, we conclude that the estimation of $u$ is better than $10^{-12} \mathrm{~m}$ for double and $10^{-19} \mathrm{~m}$ for quad precision (as noted earlier, this holds for all methods). On the other hand, comparing the results of $\max |\delta \beta|, \max |\delta \lambda|$ and $\max \delta r$, we conclude that only quad precision provides results suitable for most practical applications. 
Table 2. Performance of the exact analytical method of Section 2.1., in the case of the Earth, using double and quad precision

\begin{tabular}{|c|c|c|c|c|c|c|c|c|}
\hline \multirow{2}{*}{ Group } & \multicolumn{5}{|c|}{ double } & \multicolumn{5}{c|}{ quad } \\
\cline { 2 - 9 } & $\max |\delta u|(\mathrm{m})$ & $\max |\delta \beta|(")$ & $\max |\delta \lambda|(")$ & $\max \delta r(\mathrm{~m})$ & $\max |\delta u|(\mathrm{m})$ & $\max |\delta \beta|(")$ & $\max |\delta \lambda|(")$ & $\max \delta r(\mathrm{~m})$ \\
\hline 1 & $9.09 \cdot 10^{-13}$ & $6.76 \cdot 10^{-7}$ & $1.40 \cdot 10^{-4}$ & $8.97 \cdot 10^{-4}$ & $4.64 \cdot 10^{-20}$ & $1.20 \cdot 10^{-21}$ & $1.36 \cdot 10^{-19}$ & $8.75 \cdot 10^{-19}$ \\
\hline 2 & $9.09 \cdot 10^{-13}$ & $1.61 \cdot 10^{-2}$ & $1.83 \cdot 10^{-1}$ & 11.3 & $3.99 \cdot 10^{-20}$ & $6.88 \cdot 10^{-10}$ & $4.79 \cdot 10^{-9}$ & $2.96 \cdot 10^{-7}$ \\
\hline 3 & $9.09 \cdot 10^{-13}$ & 40.1 & 699 & 18.2 & $4.99 \cdot 10^{-20}$ & $9.78 \cdot 10^{-3}$ & $1.71 \cdot 10^{-1}$ & $6.71 \cdot 10^{-7}$ \\
\hline 4 & $9.09 \cdot 10^{-13}$ & 2.12 & 1.24 & 13.9 & $4.90 \cdot 10^{-20}$ & $7.17 \cdot 10^{-8}$ & $1.47 \cdot 10^{-7}$ & $7.36 \cdot 10^{-7}$ \\
\hline 5 & $9.09 \cdot 10^{-13}$ & $2.54 \cdot 10^{-7}$ & 1.66 & 16.1 & $4.14 \cdot 10^{-20}$ & $8.71 \cdot 10^{-22}$ & $1.00 \cdot 10^{-7}$ & $7.04 \cdot 10^{-7}$ \\
\hline 6 & $9.09 \cdot 10^{-13}$ & $1.61 \cdot 10^{-2}$ & $2.83 \cdot 10^{-1}$ & 17.4 & $4.98 \cdot 10^{-20}$ & $6.06 \cdot 10^{-10}$ & $1.29 \cdot 10^{-8}$ & $7.94 \cdot 10^{-7}$ \\
\hline 7 & $9.09 \cdot 10^{-13}$ & 40.1 & 699 & 18.2 & $4.99 \cdot 10^{-20}$ & $1.16 \cdot 10^{-2}$ & $1.99 \cdot 10^{-1}$ & $7.72 \cdot 10^{-7}$ \\
\hline 8 & $9.09 \cdot 10^{-13}$ & 2.25 & 4.42 & 17.8 & $4.97 \cdot 10^{-20}$ & $1.14 \cdot 10^{-7}$ & $1.78 \cdot 10^{-7}$ & $7.20 \cdot 10^{-7}$ \\
\hline 9 & $9.09 \cdot 10^{-13}$ & $3.83 \cdot 10^{-7}$ & 1.66 & 19.8 & $4.93 \cdot 10^{-20}$ & $1.06 \cdot 10^{-21}$ & $9.97 \cdot 10^{-8}$ & $7.63 \cdot 10^{-7}$ \\
\hline 10 & $9.09 \cdot 10^{-13}$ & $1.44 \cdot 10^{-2}$ & $3.07 \cdot 10^{-1}$ & 19.0 & $4.97 \cdot 10^{-20}$ & $6.75 \cdot 10^{-10}$ & $1.28 \cdot 10^{-8}$ & $7.91 \cdot 10^{-7}$ \\
\hline 11 & $9.09 \cdot 10^{-13}$ & $1.47 \cdot 10^{-2}$ & $2.86 \cdot 10^{-1}$ & 17.6 & $5.00 \cdot 10^{-20}$ & $6.57 \cdot 10^{-10}$ & $1.10 \cdot 10^{-8}$ & $6.79 \cdot 10^{-7}$ \\
\hline 12 & $9.09 \cdot 10^{-13}$ & $2.42 \cdot 10^{-1}$ & 4.42 & 20.4 & $5.00 \cdot 10^{-20}$ & $1.32 \cdot 10^{-8}$ & $2.13 \cdot 10^{-7}$ & $8.63 \cdot 10^{-7}$ \\
\hline 13 & $9.09 \cdot 10^{-13}$ & 54.8 & 920 & 16.7 & $4.98 \cdot 10^{-20}$ & $1.16 \cdot 10^{-2}$ & $1.99 \cdot 10^{-1}$ & $7.16 \cdot 10^{-7}$ \\
\hline
\end{tabular}

We overemphasize that, the ellipsoidal coordinates are valuable in gravity field related studies and taking into account the current estimates of the related quantities, a precision better than a millimeter is sufficient.

Comparing the results of $\max |\delta \beta|, \max |\delta \lambda|$ and max $\delta r$ presented in Table 3 between the double and quad precision, we conclude that both precisions can provide results suitable for most practical applications $(\delta r$ better than $1 \mathrm{~cm}$ for double and $10^{-11} \mathrm{~m}$ for quad precision, while angular coordinates better than $10^{-4}$ arcsec for double and $10^{-8}$ arcsec for quad precision).

It is worth noting that, in the exact analytical method of Section 2.2., the one coordinate is computed from the other. Therefore, the worst results are observed at the same points.

Comparing the results of $\max |\delta \beta|, \max |\delta \lambda|$ and max $\delta r$ presented in Table 4 between the double and quad precision, we conclude that only quad precision can provide results suitable for most practical applications, excluding the $x z$-plane and the area near the umbilics.

Equations (46)-(49) may be written using as first approximate value only the ellipsoidal latitude $\beta_{0}$, i.e. we first compute $\lambda$ and then $\beta$. Also, these equations may be written using approximate values for both coordinates $\beta_{0}$ and $\lambda_{0}$, as suggested by Bektas (2015). However, in both cases the results are worse than those of Table 4.

Comparing the results of $\max |\delta \beta|, \max |\delta \lambda|$ and max $\delta r$ presented in Table 5 between the double and quad precision, we conclude that both precisions can provide results suitable for most practical applications $(\delta r$ better than $10^{-11} \mathrm{~m}$ for double and $10^{-26} \mathrm{~m}$ for quad precision, while angular coordinates better than $10^{-4}$ arcsec for double and $10^{-8}$ arcsec for quad precision).
The characteristic of the numerical method of Section 3.2. is that the coordinates $\beta$ and $\lambda$ are computed independently. Therefore, the worst results are usually observed at different points.
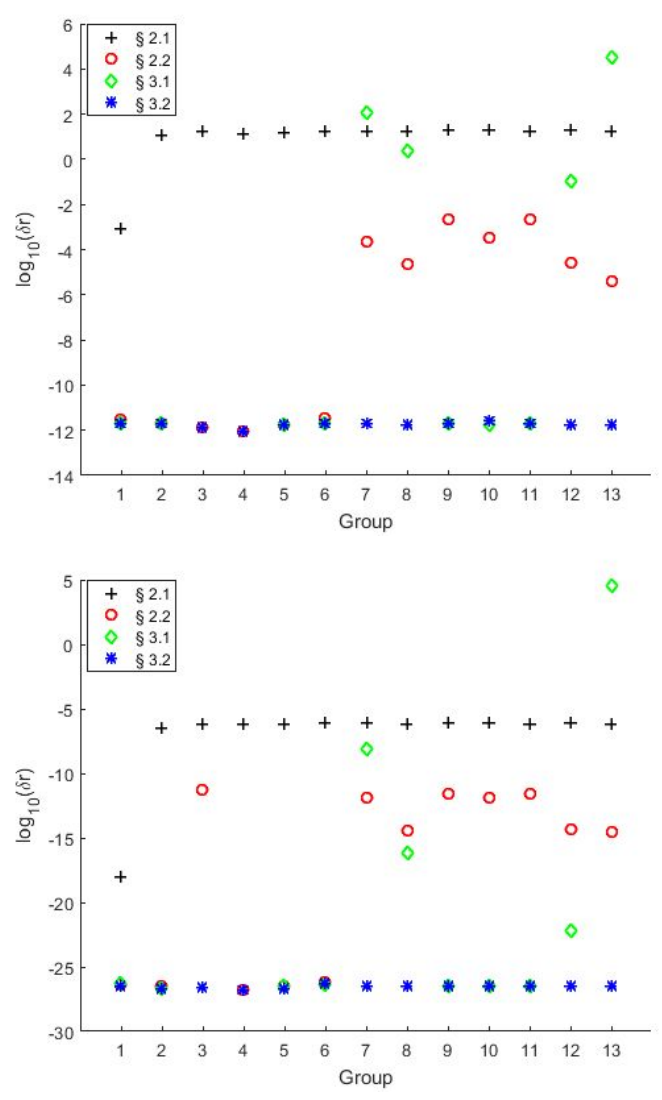

Fig. 1. Values of max $\delta$ in $\log _{10}$ scale for every group and method in: (top) double precision (bottom) quad precision 
Table 3. Performance of the exact analytical method of Section 2.2., in the case of the Earth, using double and quad precision

\begin{tabular}{|c|c|c|c|c|c|c|}
\hline \multirow{2}{*}{ Group } & \multicolumn{5}{|c|}{ double } & \multicolumn{3}{c|}{ quad } \\
\cline { 2 - 7 } & $\max |\delta \beta|(")$ & $\max |\delta \lambda|(")$ & $\max \delta r(\mathrm{~m})$ & $\max |\delta \beta|(")$ & $\max |\delta \lambda|(")$ & $\max \delta r(\mathrm{~m})$ \\
\hline 1 & $3.75 \cdot 10^{-14}$ & $5.00 \cdot 10^{-14}$ & $3.19 \cdot 10^{-12}$ & $4.78 \cdot 10^{-22}$ & $5.65 \cdot 10^{-22}$ & $4.57 \cdot 10^{-27}$ \\
\hline 2 & 0 & $2.65 \cdot 10^{-14}$ & $1.88 \cdot 10^{-12}$ & 0 & $3.80 \cdot 10^{-22}$ & $3.23 \cdot 10^{-27}$ \\
\hline 3 & $4.24 \cdot 10^{-6}$ & 0 & $1.29 \cdot 10^{-12}$ & $6.95 \cdot 10^{-10}$ & $8.82 \cdot 10^{-9}$ & $5.78 \cdot 10^{-12}$ \\
\hline 4 & 0 & $1.95 \cdot 10^{-13}$ & $9.58 \cdot 10^{-13}$ & 0 & $1.06 \cdot 10^{-20}$ & $1.68 \cdot 10^{-27}$ \\
\hline 5 & $2.50 \cdot 10^{-14}$ & 0 & $1.85 \cdot 10^{-12}$ & $3.63 \cdot 10^{-22}$ & 0 & $3.24 \cdot 10^{-27}$ \\
\hline 6 & $1.17 \cdot 10^{-15}$ & $5.00 \cdot 10^{-14}$ & $3.28 \cdot 10^{-12}$ & $1.82 \cdot 10^{-23}$ & $4.39 \cdot 10^{-22}$ & $6.46 \cdot 10^{-27}$ \\
\hline 7 & $4.24 \cdot 10^{-6}$ & $3.60 \cdot 10^{-5}$ & $2.22 \cdot 10^{-4}$ & $6.95 \cdot 10^{-10}$ & $8.79 \cdot 10^{-9}$ & $1.17 \cdot 10^{-12}$ \\
\hline 8 & $3.60 \cdot 10^{-7}$ & $2.65 \cdot 10^{-13}$ & $2.22 \cdot 10^{-5}$ & $6.66 \cdot 10^{-17}$ & $1.06 \cdot 10^{-20}$ & $4.09 \cdot 10^{-15}$ \\
\hline 9 & $2.50 \cdot 10^{-14}$ & $3.60 \cdot 10^{-5}$ & $2.21 \cdot 10^{-3}$ & $4.42 \cdot 10^{-22}$ & $3.79 \cdot 10^{-14}$ & $2.33 \cdot 10^{-12}$ \\
\hline 10 & $3.60 \cdot 10^{-6}$ & $3.60 \cdot 10^{-6}$ & $3.14 \cdot 10^{-4}$ & $9.51 \cdot 10^{-15}$ & $1.89 \cdot 10^{-14}$ & $1.17 \cdot 10^{-12}$ \\
\hline 11 & $3.90 \cdot 10^{-16}$ & $3.60 \cdot 10^{-5}$ & $2.22 \cdot 10^{-3}$ & $1.87 \cdot 10^{-23}$ & $3.79 \cdot 10^{-14}$ & $2.34 \cdot 10^{-12}$ \\
\hline 12 & $3.60 \cdot 10^{-7}$ & $3.60 \cdot 10^{-6}$ & $2.56 \cdot 10^{-5}$ & $6.66 \cdot 10^{-17}$ & $6.66 \cdot 10^{-16}$ & $4.73 \cdot 10^{-15}$ \\
\hline 13 & $4.24 \cdot 10^{-6}$ & $3.60 \cdot 10^{-5}$ & $3.88 \cdot 10^{-6}$ & $6.59 \cdot 10^{-10}$ & $8.79 \cdot 10^{-9}$ & $2.58 \cdot 10^{-15}$ \\
\hline
\end{tabular}

Table 4. Performance of the numerical method (generalized Bektas) of Section 3.1., in the case of the Earth, using double and quad precision

\begin{tabular}{|c|c|c|c|c|c|c|c|c|}
\hline \multirow{2}{*}{ Group } & \multicolumn{3}{|c|}{ double } & \multicolumn{3}{c|}{ quad } \\
\cline { 2 - 8 } & $\max |\delta \beta|(")$ & $\max |\delta \lambda|(")$ & $\max \delta r(\mathrm{~m})$ & $\begin{array}{c}\operatorname{mean}(i) \\
/ \max (i)\end{array}$ & $\max |\delta \beta|(")$ & $\max |\delta \lambda|(")$ & $\max \delta r(\mathrm{~m})$ & $\begin{array}{c}\operatorname{mean}(i) \\
/ \max (i)\end{array}$ \\
\hline 1 & $2.50 \cdot 10^{-14}$ & $5.00 \cdot 10^{-14}$ & $2.03 \cdot 10^{-12}$ & $8 / 100$ & $4.78 \cdot 10^{-22}$ & $5.65 \cdot 10^{-22}$ & $4.91 \cdot 10^{-27}$ & $10 / 150$ \\
\hline 2 & 0 & $2.50 \cdot 10^{-14}$ & $1.88 \cdot 10^{-12}$ & $2 / 2$ & 0 & $3.80 \cdot 10^{-22}$ & $2.28 \cdot 10^{-27}$ & $2 / 2$ \\
\hline 3 & - & - & - & - & - & - & - \\
\hline 4 & - & - & - & - & - & - & - \\
\hline 5 & $2.50 \cdot 10^{-14}$ & 0 & $1.85 \cdot 10^{-12}$ & $3 / 3$ & $3.63 \cdot 10^{-22}$ & 0 & $3.24 \cdot 10^{-27}$ & $3 / 3$ \\
\hline 6 & $3.90 \cdot 10^{-16}$ & $2.50 \cdot 10^{-14}$ & $2.03 \cdot 10^{-12}$ & $2 / 4$ & $1.82 \cdot 10^{-23}$ & $4.39 \cdot 10^{-22}$ & $4.04 \cdot 10^{-27}$ & $2 / 4$ \\
\hline 7 & $2.50 \cdot 10^{-14}$ & 3600 & 111 & $2 / 100$ & $5.11 \cdot 10^{-22}$ & $1.62 \cdot 10^{-2}$ & $7.74 \cdot 10^{-9}$ & $22 / 150$ \\
\hline 8 & $1.15 \cdot 10^{-4}$ & $8.62 \cdot 10^{-1}$ & 2.34 & $2 / 100$ & $6.96 \cdot 10^{-19}$ & $1.98 \cdot 10^{-16}$ & $6.09 \cdot 10^{-17}$ & $19 / 150$ \\
\hline 9 & $2.50 \cdot 10^{-14}$ & $2.50 \cdot 10^{-14}$ & $2.10 \cdot 10^{-12}$ & $3 / 4$ & $4.42 \cdot 10^{-22}$ & $1.62 \cdot 10^{-23}$ & $3.66 \cdot 10^{-27}$ & $3 / 4$ \\
\hline 10 & $1.14 \cdot 10^{-14}$ & $3.90 \cdot 10^{-16}$ & $1.85 \cdot 10^{-12}$ & $4 / 8$ & $1.47 \cdot 10^{-23}$ & $1.90 \cdot 10^{-23}$ & $3.32 \cdot 10^{-27}$ & $4 / 9$ \\
\hline 11 & $3.90 \cdot 10^{-16}$ & $2.50 \cdot 10^{-14}$ & $2.07 \cdot 10^{-12}$ & $2 / 3$ & $1.87 \cdot 10^{-23}$ & $1.50 \cdot 10^{-23}$ & $3.55 \cdot 10^{-27}$ & $2 / 3$ \\
\hline 12 & $2.50 \cdot 10^{-14}$ & $3.01 \cdot 10^{-2}$ & $1.06 \cdot 10^{-1}$ & $2 / 5$ & $1.62 \cdot 10^{-23}$ & $2.00 \cdot 10^{-23}$ & $6.36 \cdot 10^{-23}$ & $2 / 5$ \\
\hline 13 & 3600 & 919 & 33067 & $4 / 100$ & 3262 & $1.99 \cdot 10^{-1}$ & 32769 & $127 / 150$ \\
\hline
\end{tabular}

Comparing the results of all methods of conversion, as they are presented in Figure 1, we conclude that only the exact analytical method of Section 2.2. and the numerical method (Panou and Korakitis) of Section 3.2. are adequate to provide excellent results for both precisions. The basic difference is that, in the numerical method (Table 5), the worst results are observed in different points, as indicated by the values of $\max \delta r$, compared with the corresponding values in the analytical method (Table 3).

In Figure 2, we present the values of $\max \delta r$ for the group 14 of all methods of conversion in the case of the Earth. We observe that there is a small increase in $\max \delta r$ with increasing $u$ for all methods, except the exact analytical method of Section 2.1., which shows a much bigger deterioration of accuracy with increasing $u$.
As a novel method, we also investigated the performance of the exact analytical method of Section 2.2. for the two other bodies described in Section 4.1. and the results are presented in Tables 6 and 7 .

In the case of an almost oblate spheroid (Table 6), the results of $\max |\delta u|$ are better than $1 \cdot 10^{-12} \mathrm{~m}$ for double and $5 \cdot 10^{-20} \mathrm{~m}$ for quad precision. In this case, the umbilics are near the $z$-axis, where the longitude is singular.

In the case of the Moon (Table 7), the results of $\max |\delta u|$ are better than $3 \cdot 10^{-13} \mathrm{~m}$ for double and $7 \cdot 10^{-21} \mathrm{~m}$ for quad precision.

Comparing the results of the analytical method of Section 2.2. for the three bodies, we observe that this method provides almost equivalent results for the three bodies, ex- 
Table 5. Performance of the numerical method (generalized Panou and Korakitis) of Section 3.2., in the case of the Earth, using double and quad precision

\begin{tabular}{|c|c|c|c|c|c|c|c|c|}
\hline \multirow{2}{*}{ Group } & \multicolumn{5}{|c}{ double } & \multicolumn{4}{|c|}{ quad } \\
\cline { 2 - 9 } & $\max |\delta \beta|(")$ & $\max |\delta \lambda|(")$ & $\max \delta r(\mathrm{~m})$ & $\begin{array}{c}\operatorname{man}(i) \\
/ \max (i)\end{array}$ & $\max |\delta \beta|(")$ & $\max |\delta \lambda|(")$ & $\max \delta r(\mathrm{~m})$ & $\begin{array}{c}\operatorname{mean}(i) \\
/ \max (i)\end{array}$ \\
\hline 1 & $2.50 \cdot 10^{-14}$ & $2.50 \cdot 10^{-14}$ & $1.95 \cdot 10^{-12}$ & $6 / 30$ & $4.78 \cdot 10^{-22}$ & $5.65 \cdot 10^{-22}$ & $3.64 \cdot 10^{-27}$ & $17 / 30$ \\
\hline 2 & 0 & $1.25 \cdot 10^{-14}$ & $1.88 \cdot 10^{-12}$ & $4 / 30$ & 0 & $3.80 \cdot 10^{-22}$ & $2.28 \cdot 10^{-27}$ & $11 / 30$ \\
\hline 3 & $5.17 \cdot 10^{-6}$ & $7.70 \cdot 10^{-5}$ & $1.29 \cdot 10^{-12}$ & $5 / 30$ & $6.95 \cdot 10^{-10}$ & $8.82 \cdot 10^{-9}$ & $2.42 \cdot 10^{-27}$ & $12 / 30$ \\
\hline 4 & 0 & $1.06 \cdot 10^{-13}$ & $9.58 \cdot 10^{-13}$ & $6 / 30$ & 0 & $1.06 \cdot 10^{-20}$ & $1.70 \cdot 10^{-27}$ & $14 / 30$ \\
\hline 5 & $1.25 \cdot 10^{-14}$ & 0 & $1.85 \cdot 10^{-12}$ & $4 / 30$ & $3.63 \cdot 10^{-22}$ & 0 & $1.87 \cdot 10^{-27}$ & $11 / 30$ \\
\hline 6 & $3.90 \cdot 10^{-16}$ & $2.50 \cdot 10^{-14}$ & $2.03 \cdot 10^{-12}$ & $4 / 30$ & $1.82 \cdot 10^{-23}$ & $4.39 \cdot 10^{-22}$ & $4.86 \cdot 10^{-27}$ & $10 / 30$ \\
\hline 7 & $5.17 \cdot 10^{-6}$ & $7.70 \cdot 10^{-5}$ & $2.03 \cdot 10^{-12}$ & $5 / 30$ & $6.95 \cdot 10^{-10}$ & $8.79 \cdot 10^{-9}$ & $3.61 \cdot 10^{-27}$ & $11 / 30$ \\
\hline 8 & $2.50 \cdot 10^{-14}$ & $4.20 \cdot 10^{-13}$ & $1.83 \cdot 10^{-12}$ & $6 / 30$ & $1.85 \cdot 10^{-22}$ & $1.06 \cdot 10^{-20}$ & $3.33 \cdot 10^{-27}$ & $19 / 30$ \\
\hline 9 & $2.50 \cdot 10^{-14}$ & $2.50 \cdot 10^{-14}$ & $2.07 \cdot 10^{-12}$ & $4 / 30$ & $4.42 \cdot 10^{-22}$ & $1.62 \cdot 10^{-23}$ & $3.61 \cdot 10^{-27}$ & $15 / 30$ \\
\hline 10 & $3.90 \cdot 10^{-16}$ & $7.81 \cdot 10^{-16}$ & $2.73 \cdot 10^{-12}$ & $2 / 3$ & $1.47 \cdot 10^{-23}$ & $1.90 \cdot 10^{-23}$ & $3.23 \cdot 10^{-27}$ & $2 / 3$ \\
\hline 11 & $7.81 \cdot 10^{-16}$ & $2.50 \cdot 10^{-14}$ & $1.92 \cdot 10^{-12}$ & $2 / 3$ & $1.87 \cdot 10^{-23}$ & $1.50 \cdot 10^{-23}$ & $3.39 \cdot 10^{-27}$ & $8 / 30$ \\
\hline 12 & $2.50 \cdot 10^{-14}$ & $2.50 \cdot 10^{-14}$ & $1.83 \cdot 10^{-12}$ & $3 / 3$ & $1.62 \cdot 10^{-23}$ & $2.45 \cdot 10^{-23}$ & $3.43 \cdot 10^{-27}$ & $12 / 30$ \\
\hline 13 & $5.17 \cdot 10^{-6}$ & $7.70 \cdot 10^{-5}$ & $1.82 \cdot 10^{-12}$ & $2 / 30$ & $6.60 \cdot 10^{-10}$ & $8.79 \cdot 10^{-9}$ & $3.25 \cdot 10^{-27}$ & $21 / 30$ \\
\hline
\end{tabular}

Table 6. Performance of the exact analytical method of Section 2.2., in the case of an almost oblate spheroid, using double and quad precision

\begin{tabular}{|c|c|c|c|c|c|c|}
\hline \multirow{2}{*}{ Group } & \multicolumn{5}{|c|}{ double } & \multicolumn{3}{c|}{ quad } \\
\cline { 2 - 7 } & $\max |\delta \beta|(")$ & $\max |\delta \lambda|(")$ & $\max \delta r(\mathrm{~m})$ & $\max |\delta \beta|(")$ & $\max |\delta \lambda|(")$ & $\max \delta r(\mathrm{~m})$ \\
\hline 1 & $2.65 \cdot 10^{-12}$ & $1.52 \cdot 10^{-11}$ & $2.67 \cdot 10^{-12}$ & $4.79 \cdot 10^{-22}$ & $5.99 \cdot 10^{-22}$ & $6.27 \cdot 10^{-27}$ \\
\hline 2 & 0 & $2.50 \cdot 10^{-14}$ & $1.74 \cdot 10^{-12}$ & 0 & $3.87 \cdot 10^{-22}$ & $2.42 \cdot 10^{-27}$ \\
\hline 3 & $2.65 \cdot 10^{-12}$ & 1.43 & $1.29 \cdot 10^{-12}$ & $1.70 \cdot 10^{-12}$ & $1.48 \cdot 10^{-8}$ & $4.04 \cdot 10^{-27}$ \\
\hline 4 & 0 & $5.67 \cdot 10^{-5}$ & $9.63 \cdot 10^{-13}$ & $2.47 \cdot 10^{-16}$ & $7.72 \cdot 10^{-21}$ & $2.73 \cdot 10^{-15}$ \\
\hline 5 & $2.50 \cdot 10^{-14}$ & 0 & $1.29 \cdot 10^{-12}$ & $3.47 \cdot 10^{-22}$ & 0 & $2.02 \cdot 10^{-27}$ \\
\hline 6 & $1.13 \cdot 10^{-14}$ & $4.50 \cdot 10^{-14}$ & $2.61 \cdot 10^{-12}$ & $1.74 \cdot 10^{-23}$ & $4.33 \cdot 10^{-22}$ & $6.26 \cdot 10^{-27}$ \\
\hline 7 & $2.65 \cdot 10^{-12}$ & 1.43 & $2.22 \cdot 10^{-4}$ & $1.70 \cdot 10^{-12}$ & $1.47 \cdot 10^{-8}$ & $1.16 \cdot 10^{-12}$ \\
\hline 8 & $6.09 \cdot 10^{-9}$ & $5.67 \cdot 10^{-5}$ & $2.22 \cdot 10^{-7}$ & $1.53 \cdot 10^{-21}$ & $1.28 \cdot 10^{-20}$ & $9.11 \cdot 10^{-20}$ \\
\hline 9 & $2.50 \cdot 10^{-14}$ & $3.60 \cdot 10^{-5}$ & $2.21 \cdot 10^{-3}$ & $4.54 \cdot 10^{-22}$ & $3.79 \cdot 10^{-14}$ & $2.20 \cdot 10^{-12}$ \\
\hline 10 & $3.60 \cdot 10^{-6}$ & $3.60 \cdot 10^{-6}$ & $3.14 \cdot 10^{-4}$ & $9.51 \cdot 10^{-15}$ & $1.89 \cdot 10^{-14}$ & $1.17 \cdot 10^{-12}$ \\
\hline 11 & $3.90 \cdot 10^{-16}$ & $3.60 \cdot 10^{-5}$ & $2.22 \cdot 10^{-3}$ & $1.90 \cdot 10^{-23}$ & $3.79 \cdot 10^{-14}$ & $2.34 \cdot 10^{-12}$ \\
\hline 12 & $3.60 \cdot 10^{-9}$ & $3.60 \cdot 10^{-6}$ & $2.27 \cdot 10^{-7}$ & $1.53 \cdot 10^{-21}$ & $1.66 \cdot 10^{-19}$ & $9.44 \cdot 10^{-20}$ \\
\hline 13 & $1.76 \cdot 10^{-4}$ & 1.43 & $3.88 \cdot 10^{-6}$ & $1.62 \cdot 10^{-13}$ & $1.30 \cdot 10^{-10}$ & $2.04 \cdot 10^{-14}$ \\
\hline
\end{tabular}

hibiting a slightly better performance in the case of the Moon.

\section{Conclusions}

In this work, a theoretical and a numerical comparative assessment of four methods (two analytical and two numerical) of converting Cartesian $(x, y, z)$ to ellipsoidal coordinates $(u, \beta, \lambda)$ of a point in space have been presented. In all four methods the ellipsoidal parameter $u$ is calculated by the same formula, while the computations of el- lipsoidal latitude and longitude are different. In the first exact analytical method the formulas for such calculations are well-known but in the second exact analytical method the corresponding formulas are developed for the first time in this work. The first numerical method is a generalization of a method of Bektas (2015) and the second of a method of Panou and Korakitis (2019), in order to enable both methods to be applicable for points in space, not only on the surface of a triaxial ellipsoid.

Theoretically, the precision of the two exact analytical methods is limited only when one approaches singular points in the cumbersome expressions involved. On the other hand, numerical methods with iterative approxima- 
Table 7. Performance of the exact analytical method of Section 2.2., in the case of the Moon, using double and quad precision

\begin{tabular}{|c|c|c|c|c|c|c|}
\hline \multirow{2}{*}{ Group } & \multicolumn{5}{|c|}{ double } & \multicolumn{3}{c|}{ quad } \\
\cline { 2 - 7 } & $\max |\delta \beta|(")$ & $\max |\delta \lambda|(")$ & $\max \delta r(\mathrm{~m})$ & $\max |\delta \beta|(")$ & $\max |\delta \lambda|(")$ & $\max \delta r(\mathrm{~m})$ \\
\hline 1 & $2.50 \cdot 10^{-14}$ & $4.50 \cdot 10^{-14}$ & $5.12 \cdot 10^{-13}$ & $7.74 \cdot 10^{-22}$ & $1.49 \cdot 10^{-21}$ & $1.28 \cdot 10^{-27}$ \\
\hline 2 & 0 & $2.50 \cdot 10^{-14}$ & $3.22 \cdot 10^{-13}$ & 0 & $3.27 \cdot 10^{-22}$ & $1.18 \cdot 10^{-27}$ \\
\hline 3 & $1.70 \cdot 10^{-5}$ & 0 & $4.69 \cdot 10^{-13}$ & $2.98 \cdot 10^{-9}$ & $1.56 \cdot 10^{-8}$ & $5.96 \cdot 10^{-12}$ \\
\hline 4 & 0 & $2.60 \cdot 10^{-13}$ & $2.41 \cdot 10^{-13}$ & $3.58 \cdot 10^{-13}$ & $4.03 \cdot 10^{-21}$ & $2.31 \cdot 10^{-12}$ \\
\hline 5 & $2.50 \cdot 10^{-14}$ & 0 & $2.57 \cdot 10^{-13}$ & $3.75 \cdot 10^{-22}$ & 0 & $5.08 \cdot 10^{-28}$ \\
\hline 6 & $1.17 \cdot 10^{-15}$ & $2.65 \cdot 10^{-14}$ & $6.65 \cdot 10^{-13}$ & $1.43 \cdot 10^{-23}$ & $7.08 \cdot 10^{-22}$ & $1.86 \cdot 10^{-27}$ \\
\hline 7 & $1.70 \cdot 10^{-5}$ & $7.14 \cdot 10^{-5}$ & $1.01 \cdot 10^{-4}$ & $2.98 \cdot 10^{-9}$ & $1.56 \cdot 10^{-8}$ & $3.18 \cdot 10^{-13}$ \\
\hline 8 & $3.60 \cdot 10^{-6}$ & $2.73 \cdot 10^{-13}$ & $6.06 \cdot 10^{-5}$ & $2.40 \cdot 10^{-15}$ & $6.87 \cdot 10^{-21}$ & $3.98 \cdot 10^{-14}$ \\
\hline 9 & $2.50 \cdot 10^{-14}$ & $3.60 \cdot 10^{-5}$ & $6.04 \cdot 10^{-4}$ & $7.06 \cdot 10^{-22}$ & $3.79 \cdot 10^{-14}$ & $6.37 \cdot 10^{-13}$ \\
\hline 10 & $3.60 \cdot 10^{-6}$ & $3.60 \cdot 10^{-6}$ & $8.39 \cdot 10^{-5}$ & $9.51 \cdot 10^{-15}$ & $1.89 \cdot 10^{-14}$ & $3.19 \cdot 10^{-13}$ \\
\hline 11 & $3.90 \cdot 10^{-16}$ & $3.60 \cdot 10^{-5}$ & $6.06 \cdot 10^{-4}$ & $1.15 \cdot 10^{-23}$ & $3.79 \cdot 10^{-14}$ & $6.39 \cdot 10^{-13}$ \\
\hline 12 & $3.60 \cdot 10^{-6}$ & $3.60 \cdot 10^{-6}$ & $6.31 \cdot 10^{-5}$ & $2.40 \cdot 10^{-15}$ & $2.40 \cdot 10^{-15}$ & $4.20 \cdot 10^{-14}$ \\
\hline 13 & $1.70 \cdot 10^{-5}$ & $7.15 \cdot 10^{-5}$ & $1.06 \cdot 10^{-7}$ & $3.59 \cdot 10^{-9}$ & $1.56 \cdot 10^{-8}$ & $1.31 \cdot 10^{-17}$ \\
\hline
\end{tabular}
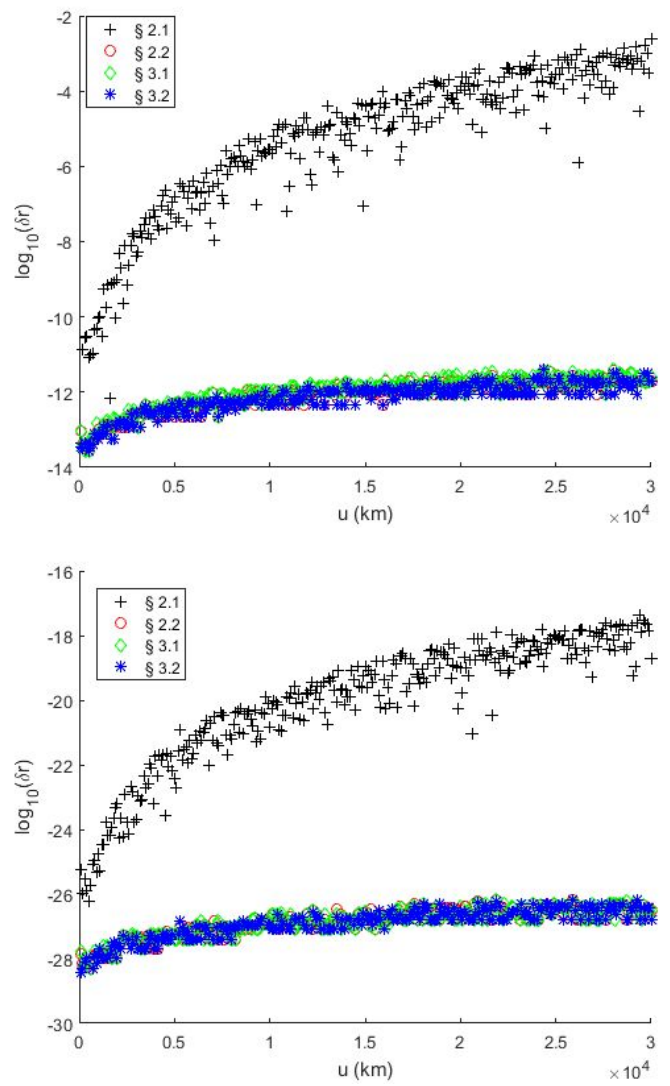

Fig. 2. Values of $m a x \delta r$ in $\log _{10}$ scale for the group 14 and all methods in: (top) double precision (bottom) quad precision

tions are more precise but the execution time is longer. Practically, all methods are validated with numerical experiments using an extensive set of test points, common for all methods.
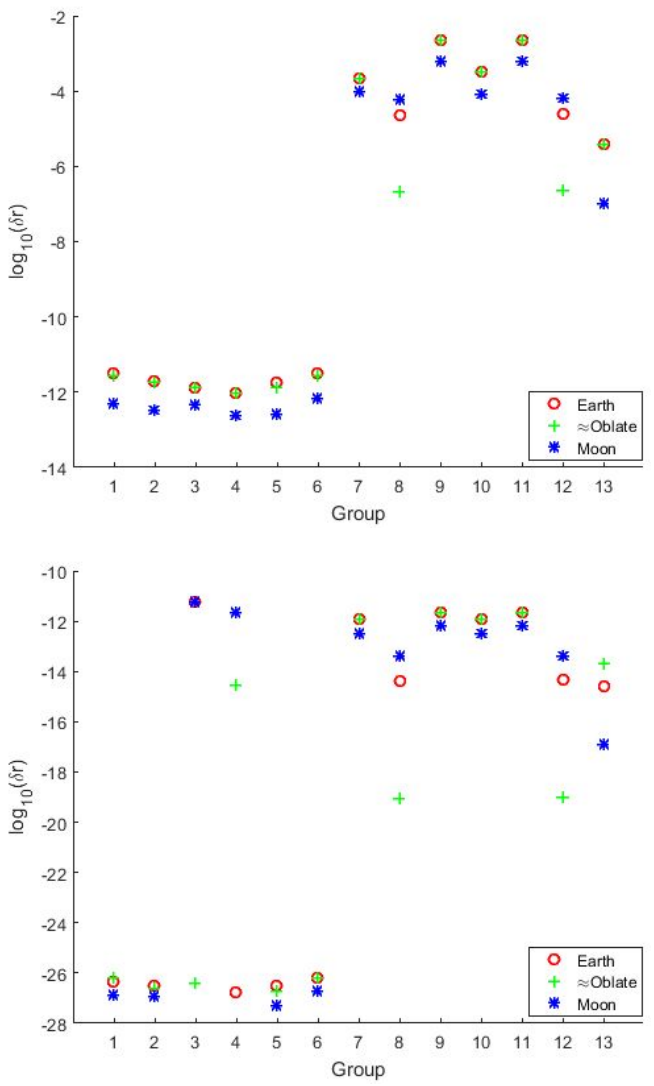

Fig. 3. Values of $\max \delta r$ in $\log _{10}$ scale for every group of the exact analytical method of Section 2.2. for the three bodies in: (top) double precision (bottom) quad precision

Comparing the results of all methods of conversion, for a test set of 7023 points for the Earth, we conclude that only the exact analytical method of Section 2.2. and the 
numerical method (Panou and Korakitis) of Section 3.2. are adequate to provide excellent results for both precisions. Specifically, the numerical validations of the analytical method produce values of $\delta r$ better than $1 \mathrm{~cm}$ for double and $10^{-11} \mathrm{~m}$ for quad precision, whereas in the numerical method the values of $\delta r$ are better than $10^{-11} \mathrm{~m}$ for double and $10^{-26} \mathrm{~m}$ for quad precision. Through the analytical method, we also have at hand formulas for the calculation of ellipsoidal latitude and longitude useful for other calculations, such as the derivatives etc., while the numerical method provides only a way of computation. Also, another difference is that, in the numerical method, the worst results for $\beta$ and $\lambda$ are observed in different points compared with the analytical method.

We also investigated the performance of all methods of conversion with respect to a wide range of values of ellipsoidal parameter $u$ (300 points for the Earth). Again, the two aforementioned methods provide reliable results. Also, as a novel exact analytical method, which can provide approximate values for the numerical method, we also analyzed its performance for two other bodies (an almost oblate spheroid and the Moon), with almost equivalent results. Finally, considering all above detailed comparisons, we conclude that the generalized Panou and Korakitis' numerical method (Section 3.2.), starting with approximate values from the new exact analytical method (Section 2.2.), is the best choice in terms of accuracy of the resulting ellipsoidal coordinates.

Acknowledgements: The authors greatly appreciate valuable suggestions and comments by anonymous referees which improved the readability of the article.

\section{References}

Bektas S., 2015. Geodetic computations on triaxial ellipsoid. International Journal of Mining Science, 1, 25-34.

Dassios G., 2012. Ellipsoidal Harmonics: Theory and Applications. Cambridge University Press, Cambridge.

Florinsky I.V., 2018. Geomorphometry on the surface of a triaxial ellipsoid: towards the solution of the problem. International Journal of Geographical Information Science, 32, 1558-1571.

Ghilani C. and Wolf P., 2006. Adjustment Computations: Spatial Data Analysis. $4^{\text {th }}$ ed., John Wiley \& Sons, Inc.

Heiskanen W.A. and Moritz H., 1967. Physical Geodesy. W.H. Freeman and Co., San Francisco and London.

Iz H. Bâki., 2009. New parameters of geometrically best fitting lunar figures. Journal of Applied Geodesy, 3, 155-162.

Jacobi C.G.J., 1839. Note von der geodätischen Linie auf einem Ellipsoid und den verschiedenen Anwendungen einer merkwürdigen analytischen Substitution. Journal für die Reine und Angewandte Mathematik, 1839, 309-313.
Panou G., 2014. A Study on Geodetic Boundary Value Problems in Ellipsoidal Geometry. Ph.D. Thesis, Department of Surveying Engineering, National Technical University of Athens, Greece.

Panou G. and Korakitis R., 2019. Geodesic equations and their numerical solution in Cartesian coordinates on a triaxial ellipsoid. Journal of Geodetic Science, 9, 1-12.

Panou G., Korakitis R. and Pantazis G., 2020. Fitting a triaxial ellipsoid to a geoid model. Journal of Geodetic Science, 10, 69-82.

Pędzich P., 2019. A low distortion conformal projection of a tri-axial ellipsoid and its application for mapping of extra-terrestrial objects. Planetary and Space Science, 178, 104697.

Tabanov M.B., 1999. Normal forms of equations of wave functions in new natural ellipsoidal coordinates. In: Uraltseva N.N. (Ed.), Proceedings of the St. Petersburg Mathematical Society, Volume V. American Mathematical Society Translations - Series 2, 193, 225-238. 